\title{
EMC3-Eirene simulations of the tungsten injection experiments in Tore Supra
}

\author{
Tilmann Lunt ${ }^{1,}$, T.Lunt ${ }^{\mathrm{a}}$, M.Kočan ${ }^{\mathrm{a}}$, J.Gunn $^{\mathrm{b}}$, Y.Feng ${ }^{\mathrm{c}}$, O.Meyer $^{\mathrm{b}}$ \\ ${ }^{a}$ Max-Planck-Institut für Plasmaphysik, Boltzmannstr. 2, 85748 Garching, Germany \\ ${ }^{b}$ CEA, IRFM, F-13108 Saint-Paul-Lez-Durance, France \\ ${ }^{c}$ Max-Planck-Institut für Plasmaphysik, Wendelsteinstr. 1, 17491 Greifswald, Germany
}

\begin{abstract}
We report on the first full 3D EMC3-Eirene simulations of the bulk plasma, neutral gas and W impurity transport in Tore Supra (TS). Three configurations are addressed, (A) the high field side-, (B) bottom- and (C) low field side limited case and compared to Mach probe measurements at the top of the device. As in previous 2D simulations the 3D modelling also showed a strong discrepancy between the measured and simulated Mach number in case B. In order to investigate the finite size of the Mach probe we included this object as a plasma limiting structure in the simulation and found that the probe indeed perturbs the plasma, which explains the discrepancy only partly. The $\mathrm{W}$ transport simulation was found to have a rather weak dependence on the magnetic configuration and the simulated tungsten confinement time shows a similar behavior as those measured recently by Meyer et al. The absolute value, however, differs by a factor of ten.
\end{abstract}

Keywords:

PACS: 52.65.Pp, 52.25.Vy, 52.55.Fa, 52.70.Ds

PSI-20: Tore Supra, Edge modeling, Tungsten, Impurity transport, Plasma flow

\section{Introduction and motivation}

Due to their high capability to withstand the enormous heat loads expected in large fusion devices like ITER and DEMO, their low sputtering yield and their low tritium

Email address: tilmann.lunt@ipp.mpg.de (Tilmann Lunt) 
retention, high $\mathrm{Z}$ materials like tungsten (W) are most likely the appropriate material for the high heat flux plasma facing components in future fusion reactors. However, the use of these materials is also challenging, because high $\mathrm{Z}$ impurities can significantly degrade the energy confinement when penetrating the hot regions of the plasma, so that only very low concentrations can be tolerated there. Several experimental investigations were performed at ASDEX Upgrade (AUG) and other machines to study the intrinsic erosion processes during normal plasma operation [2], as well as behavior of $\mathrm{W}$ from intentionally inserted sources, e.g. by melting solid $\mathrm{W}$ rods in a controlled way [3]. Since the melting dynamics, the macroscopic transport of ejected W droplets and the impurity transport in the plasma are all three complex processes, their individual contribution to the total amount of $\mathrm{W}$ in the plasma is difficult to disentangle. During the 2011 campaign defined amounts of gaseous $\mathrm{W}(\mathrm{CO})_{6}$ were injected into Tore Supra (TS) limiter plasmas at different radial positions in the scrape-off [14] layer while monitoring the core W content by VUV spectroscopy [12], which allow the study of the W impurity transport in the plasma separately. These experiments, which were motivated by the planned installation of a full $\mathrm{W}$ divertor in TS in the framework of the WEST project[1], were accompanied by simulations of the bulk plasma, neutral particle and $\mathrm{W}$ impurity transport by means of the Edge Monte Carlo 3D - Eirene (EMC3-Eirene) code package [4]. This code is suitable for this endeavor since the complex plasma limiter interaction requires full 3D treatment of the problem. Furthermore, it was already applied for similar studies at AUG $[5,6,7]$.

As a first step we present simulations of the deuterium bulk plasma limited at different poloidal positions in Sec. 2 and compare these to measurements of a fast reciprocating Mach probe and other diagnostics. Given that the probe is a rather large object (diameter $4 \mathrm{~cm}$ ) with respect to typical SOL fall-off lengths we exploit the 3D capacity of the code and treat the probe as a plasma limiting structure.

The following section 3 addresses the $\mathrm{W}$ impurity transport simulations, which are compared to the $\mathrm{W}(\mathrm{CO})_{6}$ injection experiments mentioned above. Sec. 4 closes with a summary. 


\section{EMC3-Eirene simulation of the bulk plasma}

The working principle of EMC3-Eirene as well as the construction of a field-line aligned 3D grid are explained in detail in Ref. [4] while recent applications of the code at AUG are documented in Refs. [5, 6, 7]. The code applies Monte Carlo techniques to solve the plasma fluid equations formulated by Braginskii and the kinetic equation for the neutrals.

TS is equipped with one discrete low field side ('LFL') and one toroidally symmetric bottom pump ('TPL') limiter, whose distances to the plasma can be adjusted during the discharge. The positions, where $z_{\min , T P L}$ and $R_{\min , L F L}$ represent the $z$ and the $R$ coordinates of the closest points of the surfaces to the machine axis $\left(R_{0}=2.421 \mathrm{~m}, z_{0}=0 \mathrm{~m}\right)$ of LFL and TPL respectively, are documented in Tab. 1. The LFL as well as the three ICRH ('Q1', 'Q2' and 'Q5') and two lower hybrid ('C2' and 'C3') antennae and the high field side limiter ('HFL') have circular shapes with different radii.

Three different configurations ('A', 'B' and ' $\mathrm{C}$ ') were addressed in the simulations, where the last closed flux surface (LCFS) is in contact with HFL, TPL and LFL, respectively. The computational grids for these configurations, which were constructed from EFIT [8] equilibria, are shown in the top line of Fig. 1. The dark red lines represent the grid for the plasma, while light red regions correspond to that for the neutrals only. The rows below show simulated $n_{e}, M$ and $T_{e}$ profiles compared to those measured by the vertically scanning Mach probe located at $R=2.528 \mathrm{~m}$. The transport coefficients (constant over the computational domain) as well as the assumed densities at the LCFS are given in the figure, while the the input power $P_{\text {in }}=P_{\text {heat }}-P_{\text {rad }}$ is documented in Tab. 1.

In order to approximate the experimental profiles and in particular to explain the high densities measured $>60 \mathrm{~mm}$ away from the LCFS, particle diffusion coefficients as large as $D=5.0 \mathrm{~m}^{2} / \mathrm{s}$ have to be assumed in case A. Despite the strong discrepancy with the probe measurements shown by the dotted, green line in Fig. 1 we repeated the simulation with the same particle transport coefficient of $D=0.25 \mathrm{~m}^{2} / \mathrm{s}$ as in configuration $\mathrm{B}$ and $\mathrm{C}$ in order to compare the three distinguishing between transport and 
geometry effects. We will also refer to this simulation as case A'.

While the Mach number measured by the probe shows at least the same sign as the simulated ones in configuration A and C, a strong discrepancy was found in case B, where the code predicts values in the range $M=-0.1 \ldots 0.2$, while the probe measures $M \lesssim-0.5$. This discrepancy was found earlier in TS [9] and JET [10, 11] comparing the profiles to $2 \mathrm{D}$ code simulations. Drifts could only partly explain the experimental results so that a ballooning like transport was assumed to be important there. Questions also arose whether the probe itself could perturb the plasma, an issue which can now be addressed by means of the 3D code EMC3-Eirene. We repeated the simulation including a virtual probe as a plasma limiting structure $25 \mathrm{~mm}$ away from the LCFS (cf. black bar in the figure) computing the particle flux to the two sides of the object. As shown by the black crosses in Fig. 1 the Mach numbers evaluated according to Hutchinson's formula [13], $M=0.4 \ln \left(j_{\text {up }} / j_{\text {down }}\right)$, are clearly lower than those of the unperturbed plasma. Although the effect points into the right direction it cannot explain fully the measurement: at the position of the collector of the real probe $(40 \mathrm{~mm})$ as indicated by the highlighted data point $\mathrm{M}$ is about -0.1 instead of $\mathrm{M}=-0.6$ in the experiment (The other data points in this figure represent the hypothetical case, where the collector was at a different position along the probe and are only shown in order to point out that there is a systematic discrepancy). Due to the low collisionalty in TS the parallel viscosity in the experiment might be lower than in the simulation. In order to test this effect, we repeated the simulation with $\eta_{\|}=0$ as shown by the dotted blue line (and the blue rhombuses) in the same figure. Fig. 3 shows the Mach number on the surface $\Psi_{N}=1.053$, the surface that is hitting the probe collector. As expected from a simple estimation, the length of the volume perturbed by the probe (blue filament in the figure) is $0.5 c_{s} \Delta x^{2} / D \approx 70 \mathrm{~m}$, where $\Delta x$ is the diameter of the probe, which is of the order of the connection length. The assumption that the probe measures local quantities is therefore strongly questioned. In order to find a self-consistent agreement with the probe profiles much more work including a detailed study of the accuracy of the equilibrium, the effect of the magnetic field ripple as well as the validity of the fluid approach is required. 


\section{Tungsten transport simulations compared to $\mathrm{W}(\mathrm{CO})_{6}$ injections}

Despite the discrepancy we found in configuration B between the experiment and the simulation we perform a $\mathrm{W}$ transport simulation on that plasma background. In terms of equilibrium as well as external discharge parameters case B is the most similar to that in [12]. Since the $\mathrm{W}(\mathrm{CO})_{6}$ molecules are assumed to dissociate immediately in the plasma and the carbon and oxygen to perturb only little (if the amount of injected $\mathrm{W}(\mathrm{CO})_{6}$ was large enough that the $\mathrm{C}$ and $\mathrm{O}$ radiate significantly, the discharge would most likely not survive the radiation from $\mathrm{W}$ ), the injector can be regarded as a radially varying point source of atomic $\mathrm{W}$. We simulate this situation by placing a $\mathrm{W}$ point source of strength $\Phi_{W}$ in EMC3-Eirene, while computing the mean $\mathrm{W}$ density $\bar{n}_{W}$ in the region inside the LCFS. From this we determine the total amount of $\mathrm{W}$ in the core $N_{W}=\bar{n}_{W} \cdot V_{\text {core }}$ assuming purely diffusive transport (and therefore a flat $n_{W}$ profile) there. Dividing this number by the source strength $\Phi_{W}$ gives an impurity con-

finement time $\tau_{W}^{E M C 3}=N_{W} / \Phi_{W}$. We repeat the simulation for several positions along the trajectory of the injector nozzle and compare these in Fig. 2 (black solid curve) to the measurement of the ones of [12]. In contrast to the simulation the experimental injection is a transient event and so the confinement time was evaluated according to $\tau_{W}=\overline{n_{e}} V^{c} / N_{W}^{s} \int_{t_{1}}^{t_{2}} c_{W}(t) d t$, where $N_{W}^{s}$ is the number of $\mathrm{W}$ atoms injected in the SOL and $c_{W}$ the measured $\mathrm{W}$ core concentration. As shown in Fig. 2 the simulated $\tau_{W}^{E M C 3}$ and measured $\tau_{W}$ show a similar dependence on the injection position, $\tau_{W}$, however, is about a factor of ten larger. Given the complexity of the physical processes involved as well as the simplified assumptions about the transport in the core and the negligence of the radiation induced by the injection of $\mathrm{W}$ we cannot expect a much better result sofar. A similar dependency is found for configurations A' (blue dashed-dotted) and C (green dashed), which shows that the result does not depend sensitively on the LFS contact (and therefore a possibly incomplete description of the bulk plasma). This can be understood noticing that the transport to the core is determined by the time the impurities remain in the SOL, i.e. the sum of times $\tau_{+}$and $\tau_{-}$needed to travel towards the limiter in parallel or anti-parallel directions. Given that the connection length $L_{c}=L_{+}+L_{-}$is about the same in the three discharges, so is the sum of traveling times $\tau_{ \pm}=L_{ \pm} / c_{s, W}$, 
where $c_{s, W}$ is the speed of sound of the $\mathrm{W}$ impurities.

If the radial diffusion coefficient $D$ is as large as in case A the profile becomes significantly flatter.

\section{Summary}

The full 3D bulk plasma, neutral gas and W impurity transport in Tore Supra was simulated by means of EMC3-Eirene with spatially constant anomalous transport coefficients. Three different magnetic configurations A, B and C with the last closed flux surface (LCFS) touching the high field side (HFL)-, the toroidal pump - and the low field side (LFS) limiter were addressed. Whereas the Mach number measured by a Mach probe in the upper part of the machine showed at least the same sign as the simulated ones in configuration A and C a strong discrepancy was found in case B, where the connection length was significantly longer than in A and B. In order to test the influence of the size of the Mach probe, we included the probe as a limiting structure in the simulation and indeed found that the Mach number evaluated for the virtual probe is notably smaller than that of the unperturbed plasma. Despite the somehow incomplete description of the bulk plasma the $\mathrm{W}$ impurity transport was simulated for all three cases and compared to experimental data showing a similar dependency on the injection position, but a factor of ten difference in magnitude. 


\begin{tabular}{cccccccc}
\hline Discharge & $\begin{array}{c}\text { time } \\
{[\mathbf{s}]}\end{array}$ & $\begin{array}{c}\text { LCFS } \\
\text { contact }\end{array}$ & $\begin{array}{c}\mathbf{P}_{H} \\
{[\mathbf{k W}]}\end{array}$ & $\begin{array}{c}\mathbf{P}_{\text {rad }} \\
{[\mathbf{k W}]}\end{array}$ & $\begin{array}{c}\bar{n}_{e} \\
{\left[\mathbf{1 0}^{18} \mathbf{m}^{-2}\right]}\end{array}$ & $\begin{array}{c}z_{\text {min }, T P L} \\
{[\mathbf{m m}]}\end{array}$ & $\begin{array}{c}R_{\min , L F L} \\
{[\mathbf{m m}]}\end{array}$ \\
\hline (A) 44052 & 8.8 & HFL & 513 & 153 & 8.4 & -717 & 3119 \\
(B) 33395 & 5.2 & TPL & 980 & 745 & 11.3 & -718 & 3115 \\
(C) 33463 & 9.7 & LFL & 441 & 249 & 22.6 & -718 & 3144 \\
\hline
\end{tabular}

Table 1: Discharge parameters and transport coefficients of the three configurations. The transport coefficients as well as the assumed densities at the LCFS are given in Fig. 1.

\section{References}

[1] Bucalossi J. el al. Fusion Engin. Des. 86 (2011) 684-688

[2] Dux R. el al. J. Nucl. Mater. 390-391 (2009) 858-863

[3] Krieger K. el al. J. Nucl. Mater. 415 (2011) S297-S300

[4] Feng Y. et al. Contrib. Plasma Phys. 44 No. 1-3 (2004) 57-69

[5] Lunt T. el al. J. Nucl. Mater. 415 (2011) S505-S508

[6] Lunt T. el al. Plasma Phys. Control. Fusion 53 (2011) 125010

[7] Lunt T. el al. Nucl. Fusion 52 (2011) 054013

[8] O’Brien D.P. el al. Nucl. Fusion 32 (1992) 1351

[9] Zagorski R. el al. Plasma Phys. Control. Fusion 49 (2006) S97-S108

[10] Matthews G.F. el al. J. Nucl. Mater. 337-339 (2005) 1-9

[11] Pitts R.A. el al. J. Nucl. Mater. 337-339 (2005) 146-153

[12] Meyer O. el al. 2012 this conference

[13] Hutchinson I.H. Phys. Rev. A 11 (1988) 4358-4366

[14] Kočan M. el al. submitted to Rev. Sci. Inst. (2012)

[15] Geier A. el al. Plasma Phys. Control. Fusion 44 (2002) 2091-2100 

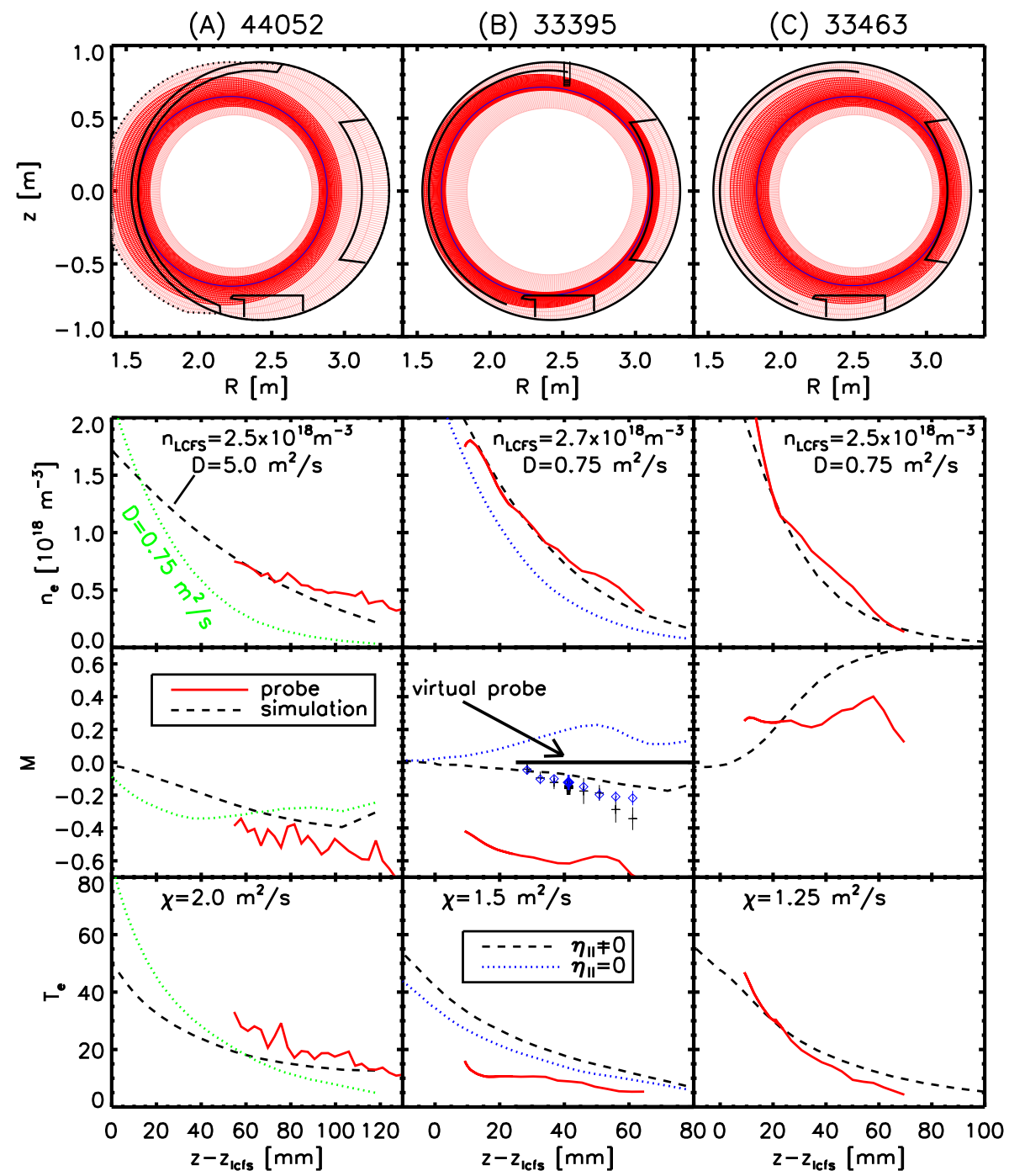

Figure 1: Left to right: Tore Supra discharges with high field side-, bottom and low field side limiter contact. Top-bottom: poloidal cross section of the computational grids, electron density, Mach number and electron temperature profiles compared to the Mach probe data. The dotted black curves represent the simulated and the solid red the measured profiles. 


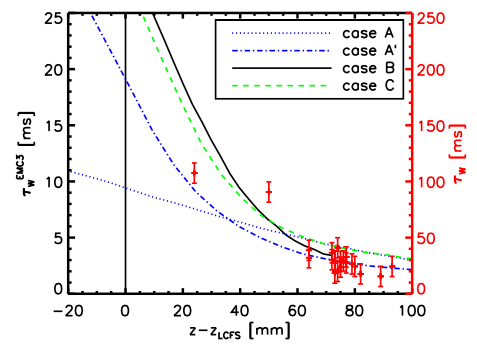

Figure 2: Simulated mean residence time for tungsten $\tau_{W}$ as a function to the injection position compared to those measured in [12].

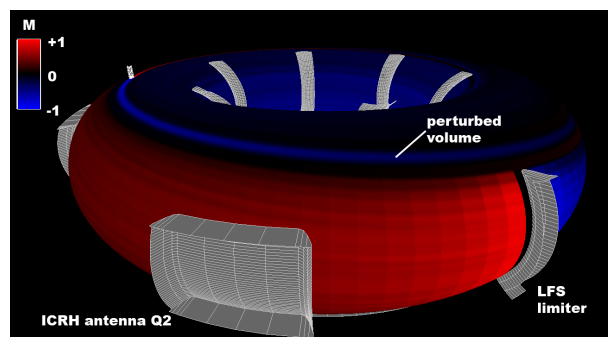

Figure 3: 3D representation of the Mach number of config B with a Mach probe located at $\phi=40^{\circ}$. The perturbed volume extends basically over the whole SOL. 